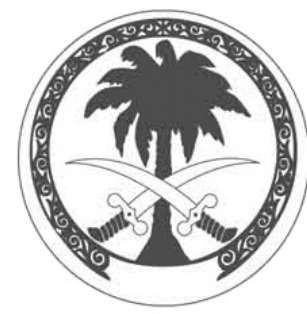

\author{
Kingdom of Saudi Arabia \\ King Faisal Specialist \\ Hospital and Research Centre - Riyadh
}

\title{
Department of Urology
}

The King Faisal Specialist Hospital and Research Centre (General Organization) (KFSH\&RC-Gen Org) is a tertiary care, state of the art Hospital situated in the cities of Riyadh and Jeddah, Kingdom of Saudi Arabia and accredited by the Joint Commission International Accreditation (JCIA). Our combined bed capacity of 1,331 is dispersed over three locations. KFSH\&RC is the national referral of sub-specialties for Oncology, Organ Transplantation, Cardiovascular Diseases, Neurosciences and Genetic Diseases with postgraduate education programs that support both Residency and Fellowship Training. Expansion projects are planned, including the development of a Pediatric Care Hospital, a dedicated Oncology \& Liver Centre and a Biotechnology Centre.

The Department of Urology in King Faisal Specialist Hospital \& Research Center is seeking a qualified Consultant, Pediatric Urologist who possess international reputation in clinical excellence and innovation to join our organization. The Consultant, Pediatric Urologist will be responsible for the diagnosis and treatment of medical diseases and conditions as well as the provision of medical care in the field of Pediatric Urology in accordance with the current medical staff regulations and the Hospital's policies and procedures. Qualified candidate(s) has to be a graduate from an accredited medical school with the completion of the required period of post graduate training in the specialty and subspecialty of Urology, Pediatric Urology, Arab or American Board certification or membership and/or Fellowship status in the United Kingdom or Canada or their equivalent is required. Candidate for Consultant, Pediatric Urology position should have a minimum experience requirement of 7 years of training in specialty or subspecialty plus post training experience is required.

\section{Please direct your electronic responses to:}

\section{E-Mail: recruitment@kfshrc.edu.sa}

\section{Employment Services- International Recruitment}

King Faisal Specialist Hospital \& Research Centre-Riyadh (MBC \#68)

Post Office Box 3354, Riyadh, 11211, Saudi Arabia Telephone: (+966-1) 442-7345

For more information about the King Faisal Specialist Hospital and Research CentreRiyadh, please visit our web site at www.kfshrc.edu.sa.

" Come for the experience ... Stay for the opportunities" 